\title{
Gate controlled Aharonov-Bohm-type oscillations from single neutral excitons in quantum rings
}

\author{
F. Ding, ${ }^{1,2,3,4, *}$ N. Akopian, ${ }^{4}$ B. Li,${ }^{5}$ U. Perinetti,${ }^{4}$ A. Govorov, ${ }^{6}$ F. M. Peeters,${ }^{5}$ C. C. Bof Bufon, ${ }^{1}$ C. Deneke, ${ }^{1}$ \\ Y. H. Chen, ${ }^{2}$ A. Rastelli, ${ }^{1}$ O. G. Schmidt, ${ }^{1}$ and V. Zwiller ${ }^{4}, \uparrow$ \\ ${ }_{1}^{1}$ Institute for Integrative Nanosciences, IFW Dresden, Helmholtzstr. 20, D-01069 Dresden, Germany \\ ${ }^{2}$ Key Laboratory of Semiconductor Materials Science, Institute of Semiconductors, Chinese Academy of Sciences, \\ P.O. Box 912, Beijing 100083, China \\ ${ }^{3}$ Max-Planck-Institut für Festkörperforschung, Heisenbergstr. 1, D-70569 Stuttgart, Germany \\ ${ }^{4}$ Kavli Institute of Nanoscience, Delft University of Technology, P.O. Box 5046, 2600 GA Delft, The Netherlands \\ ${ }^{5}$ Departement Fysica, Universiteit Antwerpen, Groenenborgerlaan 171, B-2020 Antwerpen, Belgium \\ ${ }^{6}$ Department of Physics and Astronomy, Ohio University, Athens, Ohio 45701, USA \\ (Received 14 May 2010; revised manuscript received 22 June 2010; published 11 August 2010)
}

\begin{abstract}
We report on a magnetophotoluminescence study of single self-assembled semiconductor nanorings which are fabricated by molecular-beam epitaxy combined with $\mathrm{AsBr}_{3}$ in situ etching. Oscillations in the neutral exciton radiative recombination energy and in the emission intensity are observed under an applied magnetic field. Further, we control the period of the oscillations with a gate potential that modifies the exciton confinement. We infer from the experimental results, combined with calculations, that the exciton Aharonov-Bohm effect may account for the observed effects.
\end{abstract}

DOI: 10.1103/PhysRevB.82.075309

\section{INTRODUCTION}

The Aharonov-Bohm (AB) effect allows for an experimental access to the relative quantum-mechanical phase $\Delta \varphi$ of a Schrödinger wave, which, in its absolute form $\varphi$ is a physical unobservable. ${ }^{1}$ When a charged particle (e.g., an electron) travels along some path $l$ (a singly connected region, where the magnetic field $\vec{B}=\vec{\nabla} \times \vec{A}$ is zero), its wave function acquires an additional phase $\delta \varphi=(e / \hbar) \int_{l} \vec{A} d \vec{l}$, for nonvanishing vector potential $\vec{A}$. This effect appears naturally in a ringlike structure (a multiply connected region) which can be considered as the combination of any two paths with the same end point. A variation in the encircled magnetic flux by $\Phi$ produces a phase difference (relative phase) between the two arms of the ring by $\Delta \varphi=(e / \hbar) \oint_{\text {ring }} \vec{A} d \vec{l}$ $=e \Phi / \hbar$, leading to the quantum interference between loop trajectories of opposite sense. During the 50 years since its discovery, the $\mathrm{AB}$ effect has made a significant impact on the development of physics. ${ }^{2}$ The AB effect has been observed independently for electrons and holes in microscale/ nanoscale structures. ${ }^{3-5}$ For an electron-hole pair (i.e., a neutral exciton) confined in specially designed nanostructures, the phases accumulated by the two species will be generally different after one revolution, leading to modulations between different quantum states. 6,7

Recent experimental works on the $\mathrm{AB}$ effect in ringlike semiconductor structures include magnetotransport measurements on a single-quantum ring (QR) fabricated by local oxidation with atomic force microscope (AFM) ${ }^{5}$ far-infrared spectroscopy, ${ }^{8}$ and magnetization spectroscopy ${ }^{9}$ on selfassembled InGaAs nanorings epitaxially grown by StranskiKrastanow (SK) mode. Theoretical investigations have predicted that in semiconductor quantum rings with a confined exciton, the $\mathrm{AB}$ effect can be probed from the photoluminescence (PL) emission since the change in the phase of the wave function is accompanied by a change in the exciton total angular momentum, making the PL emission magnetic field dependent. ${ }^{6,7,10-13}$ Bayer et al. ${ }^{14}$ reported this effect for
PACS number(s): 71.35.Ji, 72.80.Ey, 74.25.Ha, 74.25.Gz

a charged exciton confined in a lithography defined QR. PL emission from indirect excitons in stacked $\mathrm{ZnTe} / \mathrm{ZnSe}$ ensemble quantum dots (QDs) shows similar oscillations, ${ }^{15,16}$ this behavior was explained by the type-II band alignment which confines one carrier inside the QD and the other carrier in the barrier, mimicking a QR-like structure.

We stress that these observations are mainly governed by single charges, e.g., electrons. The AB effect can also be observed for an electron-hole pair (i.e., a neutral exciton) on condition that in a ringlike structure the electron and hole move over different paths, resulting in a nonzero electric dipole moment. ${ }^{6}$ Similar effect has been reported recently on the type-I InAs/GaAs ensemble QRs. ${ }^{17}$ However, considering the relatively small electron-hole separation, the quest for the $\mathrm{AB}$ effect in a single neutral exciton in a type-I system is more challenging. An in-plane electric field could possibly increase the polarization of an exciton and hence, the visibility of this exciton $\mathrm{AB}$ effect. ${ }^{18}$ The observation of such effect is not only of fundamental interest, Fischer et al. ${ }^{19,20}$ suggested recently that the exciton $\mathrm{AB}$ effect in a quantum ring can be dynamically controlled by a combination of magnetic and electric fields, a new proposal to trap light.

We report here on the magneto-PL study of a single selfassembled semiconductor quantum ring fabricated by in situ $\mathrm{AsBr}_{3}$ etching. ${ }^{21}$ Due to a radial asymmetry in the effective confinement for electrons and holes, we expect the neutral exciton $\mathrm{AB}$ effect. Here we present data on the AB-type oscillations in the photoluminescence energy and intensity of a single neutral exciton, which is in agreement with previous theoretical predictions. We will also show that a vertical electrical field, which modifies the exciton confinement, is able to control this quantum interference effect.

\section{EXPERIMENT}

\section{A. In situ etched In(Ga)As/GaAs quantum ring}

The sample is grown in a solid-source molecular-beam epitaxy system equipped with an $\mathrm{AsBr}_{3}$ gas source. Low 

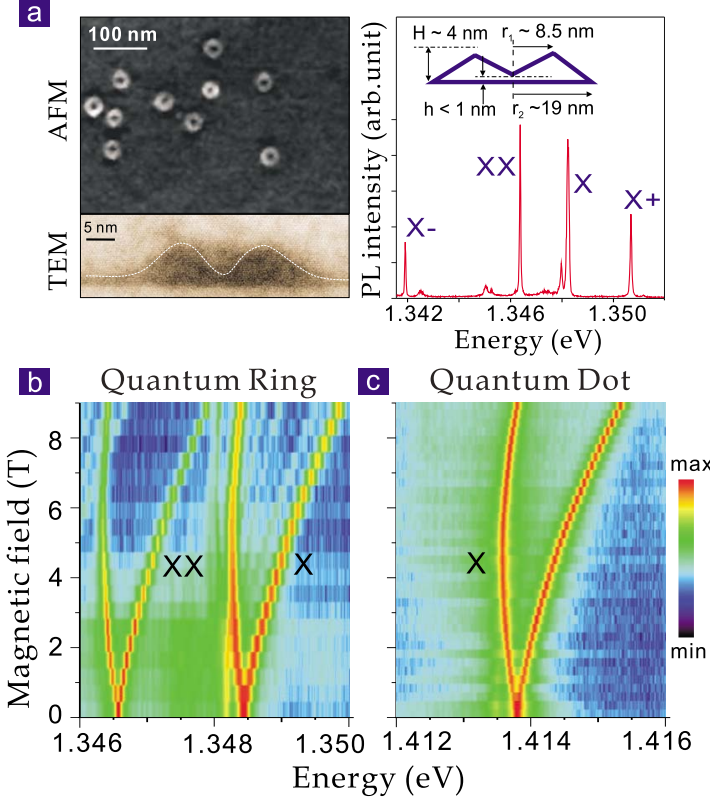

FIG. 1. (Color online) (a) AFM image of the QRs shown together with a cross-sectional TEM image of a single QR. PL spectrum of a single QR (with a nominal etching depth of $3 \mathrm{~nm}$ ) is shown in the right. The inset presents the structure parameters. [(b) and (c)] Color-coded maps of the logarithm of the PL intensity as a function of magnetic field and PL energy. For comparison, data for a single QD are shown in (c).

density SK InGaAs QDs are first grown and capped with a 10-nm-thick GaAs layer. $\mathrm{AsBr}_{3}$ etching gas is then supplied. Strain-enhanced etching results in the preferential removal of the central part of the buried QDs and the spontaneous formation of ringlike structures ${ }^{21}$ [Fig. 1(a)]. By varying the nominal etching depth, the morphology of the ring structure can be tuned. Some of the QRs are embedded in an $n$-i-Schottky structure, consisting of a $20 \mathrm{~nm} n$-doped GaAs layer followed by a 20-nm-thick spacer layer under the QRs, $30 \mathrm{~nm} i$-GaAs and a $116-\mathrm{nm}$-thick AlAs/GaAs short-period superlattice. With a semitransparent Ti top gate and a $\mathrm{Cr} / \mathrm{Au}$ alloyed back gate, a vertical electric field can be applied to modify the electrostatic potential and to control the number of electrons in the ring. ${ }^{22}$ Successive charging of a QR by up to two extra electrons is observed in our experiment.

The three-dimensional (3D) morphology of buried InGaAs QRs is observed by removal of the GaAs cap layer by selective wet chemical etching followed by AFM imaging. ${ }^{21}$ A cross-sectional transmission electron microscopy (TEM) image of a single QR is also presented [Fig. 1(a)]. For the ring studied here [nominal etching depth of $3 \mathrm{~nm}$ (Ref. 21)], the inner radius $r_{1}$ is about $8.5 \mathrm{~nm}$, an average outer radius $r_{2}$ about $19 \mathrm{~nm}$, and a height $H$ about $4 \mathrm{~nm}$. The inner height $h$ is less than $1 \mathrm{~nm}$ [inset of Fig. 1(a)].

Low-temperature PL probes the energy changes and intensity changes related to the nature of the ground state. We place the samples in a cryostat tube which is evacuated and then refilled with a small amount of helium acting as exchange gas for cooling. The tube is then inserted into a $4.2 \mathrm{~K}$ helium bath Dewar equipped with a superconducting magnet capable of providing fields up to $9 \mathrm{~T}$. This configuration guarantees extremely high mechanical stability, which is crucial for our experiment. The sample is excited by a $532 \mathrm{~nm}$ laser beam and the luminescence is collected by an objective with 0.85 numerical aperture. The PL signal is dispersed by a spectrometer with $0.75 \mathrm{~m}$ focal length equipped with a 1800 $\mathrm{g} / \mathrm{mm}$ grating, and captured by a liquid nitrogen cooled $\mathrm{Si}$ charge-coupled device (CCD).

Figure 1(a) shows a typical PL spectrum for a single QR at $B=0 \mathrm{~T}$. The neutral exciton $\mathrm{X}$, the biexciton $\mathrm{XX}$, and the charged excitons $\mathrm{X}^{-}$and $\mathrm{X}^{+}$were identified by powerdependent PL, polarization-dependent PL, and charging experiments. ${ }^{22} \mathrm{~A}$ magnetic field up to $9 \mathrm{~T}$ is applied along the sample growth direction. The evolution of a single QR PL emission with increasing $B$ is shown in Fig. 1(b). For comparison we also present data for a conventional InGaAs QD [Fig. 1(c)] with a height of about $3 \mathrm{~nm}$. Unlike for QR ensembles, ${ }^{16,17}$ the characteristic Zeeman splitting as well as the diamagnetic shift of the PL emission energy for both samples are observed, similar to previous reports on single QRs. ${ }^{23}$ A significant feature observed for the QR sample is the decrease in PL intensity with increasing magnetic field, in direct contrast to the constant PL intensity from the reference QD. The change in PL intensity is one of the signatures expected for a QR and is attributed to oscillations in the ground-state transitions. ${ }^{6,15-17}$

\section{B. Peak-determination procedure}

A well-known difficulty encountered in the magneto-PL experiments is the fact that the position of the focus laser spot changes slightly on magnetic field. The system was carefully optimized and tested to minimize drift associated with magnetic field ramping. Not only some improvements are made to increase the magneto-PL system stability but also we ramp up the field quite slowly and adjust the optics every $0.25 \mathrm{~T}$ in order to maintain the PL signal. We have increased significantly the data-acquisition time to ensure an accurate determination of the peak position and to minimize the error of the fitting procedure.

We achieve an optimum resolution of $2.5 \mu \mathrm{eV}$ by fitting the PL peaks with Lorentzian functions. This kind of analysis relies on the fact that a PL peak will usually extend over different pixels on the CCD and is widely used to determine the small fine-structure splitting (FSS) in quantum dots. ${ }^{24-26}$ We calibrate the accuracy of our peak-determination procedure by using the quantum confined Stark effect (QCSE). When a forward bias $V_{g}$ from 0.28 to $0.44 \mathrm{~V}$ (with steps of 5 $\mathrm{mV}$ ) is applied, the PL emission energy is tuned by about $120 \mu \mathrm{eV}$ [see Fig. 2(a)]. We observe that, in contrast to the discretized raw data (diamond points), the Lorentzian fit data show a smooth blueshift of the peak positions (round points). In order to evaluate the precision of this fitting method, we considered a smaller bias interval in which the Stark shift changes quadratically with the applied bias. The fitted peak position as a function of the applied bias is shown in Fig. 2(b). We can see that, as the bias is scanned in steps of 0.5 $\mathrm{mV}$ (corresponding to a Stark shift of about $0.7 \mu \mathrm{eV}$ ), the fitted peak position changes smoothly to the higher energy region and we plot the $95 \%$ prediction band of the fit func- 


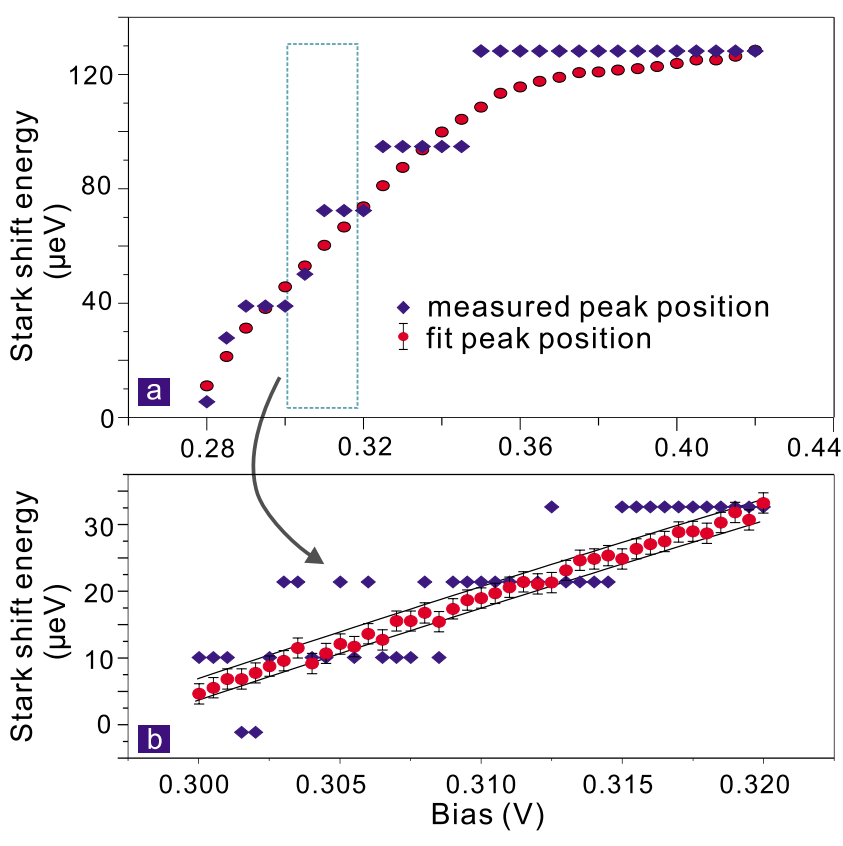

FIG. 2. (Color online) PL energy is tuned by quantum confined Stark effect at small voltage steps. The upper figure shows a large tuning range of $120 \mu \mathrm{eV}$ while the lower figure shows a smaller tuning range with much smaller voltage steps. The fit peak positions, with error of $\pm 1.5 \mu \mathrm{eV}$, fall well into the $95 \%$ prediction band of the fit function.

tion in Fig. 1(b) (solid lines), from which an error of $\pm 1.5 \mu \mathrm{eV}$ for this Lorentzian analysis procedure is estimated. For the evaluation of the systematic error, repeated measurements ( $B$ field increasing and decreasing) are performed on the same $\mathrm{QR}$ and the fit peak positions are reproducible to within $\pm 2.5 \mu \mathrm{eV}$. We can therefore expect an overall error of $\pm 2.5 \mu \mathrm{eV}$ in the peak-determination procedure used in this work (Fig. 3).

To further support the validity of this peak-determination procedure, we perform the FSS measurements for X and XX lines shown in Fig. 1. The data points are given by Lorentzian fits, with an uncertainty of $2.5 \mu \mathrm{eV}$. Solid lines in the

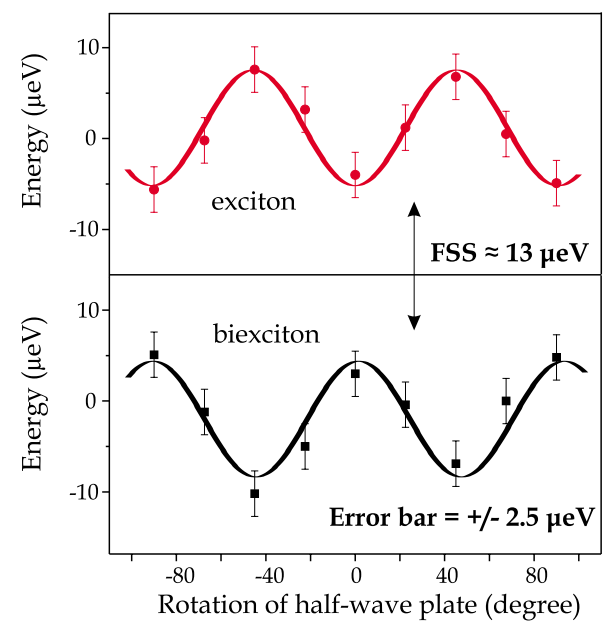

FIG. 3. (Color online) Linear polarization-dependent energy shift of $\mathrm{X}$ and XX. The solid lines are sine wave fittings.
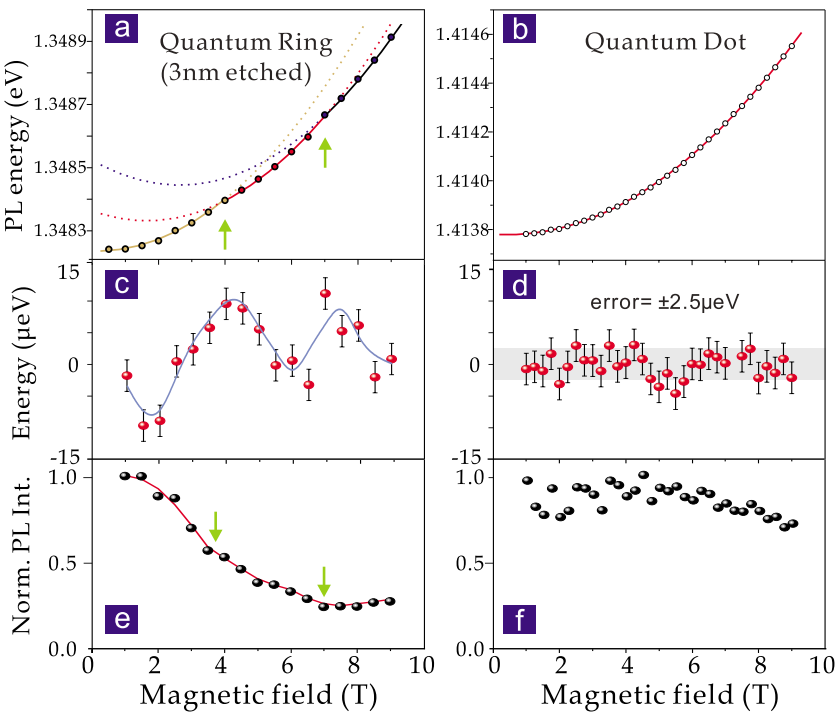

FIG. 4. (Color online) Fitted PL peak position versus $B$ field after averaging the Zeeman splitting for (a) a QR and (b) a QD neutral exciton emission. The lines are the quadratic fits. Oscillations in PL energy for the $3 \mathrm{~nm}$ etched QR and corresponding normalized PL intensity as a function of $B$ field are shown in (c) and (e), respectively. The solid lines represent the smoothed data using a Savitzky-Golay filter. For a reference QD, (d) the neutral exciton shows no energy oscillation within an error region of $\pm 2.5 \mu \mathrm{eV}$ (gray area) and (f) also the normalized PL intensity shows no clear quenching.

exciton and biexciton plots are sine wave fitting curves. As expected, $\mathrm{X}$ and $\mathrm{XX}$ show anticorrelated shifts as we rotate the half-wave plate (Fig. 3). The FSS of this QD is only $13 \mu \mathrm{eV}$, which is near the resolution limit of our PL system and would be undetectable without the Lorentzian fitting process.

\section{Magnetophotoluminescence}

The exciton emission energy in a magnetic field can be described by $E_{\mathrm{PL}}=E_{0} \pm \frac{1}{2}\left|\mathrm{~g}^{*}\right| \mu B+\gamma_{2} B^{2}$. Here $\left|\mathrm{g}^{*}\right|$ is the effective exciton Landé factor, $\mu$ is the Bohr magneton, and $\gamma_{2}$ is the diamagnetic shift coefficient. However it is much more complicated when considering an exciton confined in a quantum ring structure, the $\mathrm{AB}$ effect induced oscillations contribute to the excitonic radiative recombination energy. We plot the neutral exciton emission energies of the QR and the QD after averaging the Zeeman splitting in Figs. 4(a) and 4(b). It is observed that, for the QR, the emission energy does not scale quadratically with increasing $B$ field, instead it shows two cusps [indicated by arrows in Fig. 4(a)] (to see this clearly, we fit the data with three parabolas). From its shape, Fig. 4(a) looks quite similar to the one shown in a very recent report ${ }^{17}$ in which $\mathrm{AB}$ oscillations in ensemble InAs/GaAs QRs were observed. However, the diamagnetic shift for a single QR studied in our work is $\sim 6$ times smaller than the value for the ensemble QRs in Ref. 17, and the oscillation amplitude is much smaller. Also, no Zeeman splitting was observed in Ref. 17. 
In order to visualize the magnetic field induced oscillations, we subtract a single parabola from Fig. 4(a) and plot the results in Fig. 4(c). We observe two maxima at $\sim 4$ and $\sim 7 \mathrm{~T}$ (the solid line here, which represents the smoothed data using a Savitzky-Golay filter, is guide to the eye), suggesting changes in the ground-state transitions. In the meantime the PL intensity shows significant quenching and two knees can be observed at $\sim 4$ and $\sim 7 \mathrm{~T}$ [Fig. 4(e)]. This observation strongly contrasts with that of a conventional QD, as shown in Figs. 4(b), 4(d), and 4(f), where no oscillations in PL energy and no quenching behavior in the PL intensity are seen.

All the observations here are well above the system resolution, as discussed in Sec. II B. Especially, the PL intensity of the QR quenches by more than $70 \%$ with increasing magnetic field, which is confirmed by repeating the measurements and similar observations in other QRs. A recent study on single droplet epitaxial GaAs QR also revealed a significant reduction in the PL intensity by more than $20 \%$ at $B$ $>6 \mathrm{~T},{ }^{27}$ the exciton $\mathrm{AB}$ effect may account for the observation. We will further discuss the $B$-dependent PL intensity in Sec. III C.

\section{GATE CONTROLLED OSCILLATIONS}

\section{A. Results and discussions}

The dynamic control of the optical AB effect with an external electric field is of great interest for the creation of an exciton-based integrated circuit. ${ }^{19}$ We demonstrate this possibility with a gated $\mathrm{QR}$ structure as described in Sec. II A, see Fig. 5(a). The oscillations for a single QR under a forward bias of $2.8 \mathrm{~V}$ are clearly seen in Fig. 5(b), with the two transition points at 2.6 and $8 \mathrm{~T}$. Again, the solid line represents the smoothed data using a Savitzky-Golay filter and serves as guide to the eye. Remarkably, when the bias is decreased from 2.8 to $0.8 \mathrm{~V}$, the transition points shift smoothly to higher magnetic fields [Fig. 5(b)], indicating that the effective radii of the electron and the hole are modified by the external gate potential. The desired control over the single exciton recombination can be achieved, as we conclude from the changes in the PL intensity, by appropriate tuning of either external magnetic or electric fields [Fig. $5(\mathrm{c})]$. The vertical electrical field significantly changes the radiative recombination efficiency, which is a consequence of the quantum confined Stark effect. At $\sim 1.8 \mathrm{~V}$ the PL intensity reaches an overall maximum and then decreases. Moreover, the PL intensity is modulated by the magnetic field at each fixed electrical field, see also Fig. 8. Most interestingly, the PL intensity at $1.8 \mathrm{~V}$ first quenches by more than $25 \%$ and then recovers fully to its original value with increasing magnetic field [Fig. 5(c)].

In the following we give a comprehensive description of how the combination of external magnetic and electric field affects the behavior of a single neutral exciton confined in a QR. Although an elaborate calculation should involve the presence of a wetting layer, azimuthal anisotropy, ${ }^{28}$ and structural asymmetry, ${ }^{23}$ we model a simplified QR as depicted in Fig. 1(a) with the main aim to explain the gate controlled oscillation effect (Fig. 5). The ground-state energy
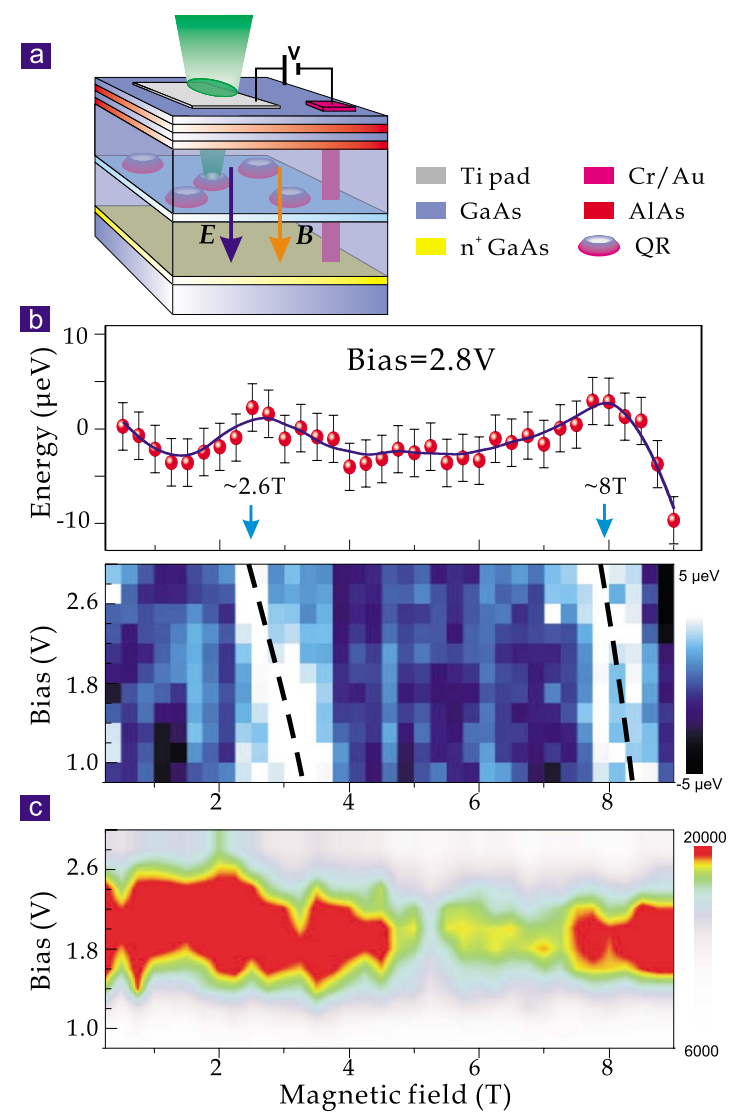

FIG. 5. (Color online) Schematic of the gated QR structure. (b) Under a forward bias of $2.8 \mathrm{~V}$ the emission energy shows clear transitions at 2.6 and $8 \mathrm{~T}$ (upper part, the solid line represents the Savitzky-Golay smoothed data and is used as guide to the eye). When the bias changes from 2.8 to $0.8 \mathrm{~V}$, the transitions shift smoothly (lower part) to higher magnetic fields. (c) Color-coded maps of the PL intensity as a function of magnetic field and external bias.

of the neutral exciton inside the $\operatorname{In}_{1-x} \mathrm{Ga}_{x} \mathrm{As}$ ring (surrounded by GaAs barrier) is calculated within the configurationinteraction method. The inner and the outer radii of the volcanolike ring are $17 \mathrm{~nm}$ and $25 \mathrm{~nm}$, respectively. While the center height of the ring is $0.5 \mathrm{~nm}$ and the top to the bottom of the ring is fixed to $5 \mathrm{~nm}$. This structure yields similar oscillations as we show in Fig. 5(b) (the actual structure parameters vary from ring to ring). The concentration of $\mathrm{Ga}$ is proportional to the distance to the bottom of the ring, which is $0.4(0.2)$ at the bottom (top) of the ring $(x=0.4$ $-0.04 z, z$ axis is the direction perpendicular to the ring plane).

We assume that only the lowest electronic subband and the highest hole band (heavy hole) is occupied. For $\mathrm{In}_{1-x} \mathrm{Ga}_{x} \mathrm{As}$, we have the effective masses $m_{e} / m_{0}=0.023$ $+0.037 x+0.003 x^{2}, m_{h} / m_{0}=0.41+0.1 x$, dielectric constant $\varepsilon$ $=\left(15.1-2.87 x+0.67 x^{2}\right) \varepsilon_{0}$, and a band gap of $E_{g}=0.36$ $+0.63 x+0.43 x^{2} \mathrm{eV}$. This results in a band-gap difference of $\Delta E_{g}=1.06-0.63 x-0.43 x^{2}$ between GaAs and $\operatorname{In}_{1-x} \mathrm{Ga}_{x} \mathrm{As}$, we take $20 \%$ of $\Delta E_{g}$ be the valence-band offset $V_{h}\left(\vec{r}_{h}\right)$ and $80 \%$ be the conduction-band offset $V_{e}\left(\vec{r}_{e}\right)$. As a simplification, we do not take the dielectric mismatch effect into ac- 
count but just assume $\varepsilon=13.8 \varepsilon_{0}$ inside and outside the quantum ring.

The full Hamiltonian of the exciton within the effectivemass approximation is given by

$$
H_{t o t}=H_{e}+H_{h}+V_{c}\left(\vec{r}_{e}-\vec{r}_{h}\right)
$$

with

$$
H_{j}=\left(\vec{P}_{j}-q_{j} \vec{A}_{j}\right) \frac{1}{2 m_{j}}\left(\vec{P}_{j}-q_{j} \vec{A}_{j}\right)+\delta E_{j}\left(\vec{r}_{j}\right)+V_{j}\left(\vec{r}_{j}\right)+q_{j} E z_{j},
$$

where the index $j=e(h)$, and $V_{j}\left(\vec{r}_{j}\right)$ is the confinement potential of the electron (hole) due to the band offset of the two materials, which is different for the inner and outer edges of the ring. $V_{c}\left(\vec{r}_{e}-\vec{r}_{h}\right)=-e^{2} / 4 \pi \varepsilon\left|\left(\vec{r}_{e}-\vec{r}_{h}\right)\right|$ is the Coulomb potential between the electron and the hole, and $\delta E_{j}\left(\vec{r}_{j}\right)$ is the strain-induced shift to the band offset which depends on the strain tensor $\epsilon_{i j}$. Here the piezoelectric potential is not taken into account since it is negligible as compared to the other terms. The last term of Eq. (1) is the potential energy in the presence of the vertical electric field $E$. Here the total angular momentum is a good quantum number and should be conserved, thus the exciton wave function with total angular momentum $L$ can be written as $\Psi_{L}\left(\vec{r}_{e}, \vec{r}_{h}\right)=\sum_{k} C_{k} \Phi_{k}\left(\vec{r}_{e}, \vec{r}_{h}\right)$, where $\Phi_{k}\left(\vec{r}_{e}, \vec{r}_{h}\right)=\psi_{n_{e}, l_{e}}\left(\vec{r}_{e}\right) \psi_{n_{h}, l_{h}}\left(\vec{r}_{h}\right)$ with $l_{e}+l_{h}=L$. By diagonalizing the obtained matrix we find the exciton energy levels and their corresponding wave functions. In order to observe the oscillation clearly, we give the results of the second derivative of the exciton total energy with respect to the magnetic field. ${ }^{7}$ The minima of this quantity corresponds to changes in the ground state, e.g., the transition points at $\sim 2.6$ and $\sim 8 \mathrm{~T}$ in Fig. 5(b).

The results for four different values of the electric field are shown in Fig. 6(a). Here we only give the results of the exciton states with $L=0$, as the calculation showed that the $L=0$ state is always the ground state and is optically active. From Fig. 6(a) we notice two oscillations within the given $B$ range. The indium composition inside the ring and the large lattice mismatch between the ring and barrier materials result in a considerably large strain, and this strain has a different effect on the electron and hole confinement potential (makes the hole confined in the top area of the ring and the electron confined in the center of the ring). The two oscillations within $B=10 \mathrm{~T}$ come from the angular momentum transitions of the main contributing single-particle basis function in the total exciton wave function. With increasing magnetic field from $B=0$ to $10 \mathrm{~T}$, the angular momentum pair $\left(l_{e}, l_{h}\right)$ in the state $\Phi_{k}\left(\vec{r}_{e}, \vec{r}_{h}\right)$ which has the largest contribution to the total wave function $\Psi_{L}\left(\vec{r}_{e}, \vec{r}_{h}\right)$ changes from $(0,0)$ to $(-1,1)$ and then from $(-1,1)$ to $(-2,2)$. The most important finding is that by decreasing the electric field from 200 to $20 \mathrm{kV} / \mathrm{cm}$, the two transitions shift to higher $B$ field (indicated by black arrows), which reproduces the shift trend in Fig. 5(b).

For a better understanding of this effect we study the effective radius of the electron (hole) $\left\langle\rho_{e}\right\rangle\left(\left\langle\rho_{h}\right\rangle\right)$ as defined by $\int \Psi_{L}^{*}\left(\vec{r}_{e}, \vec{r}_{h}\right) \rho_{e(h)} \Psi_{L}\left(\vec{r}_{e}, \vec{r}_{h}\right) d \vec{r}_{e} d \vec{r}_{h}$ (which represents the electron and hole positions inside the ring). From Fig. 6(b) we know that with decreasing the vertical electric field from 200
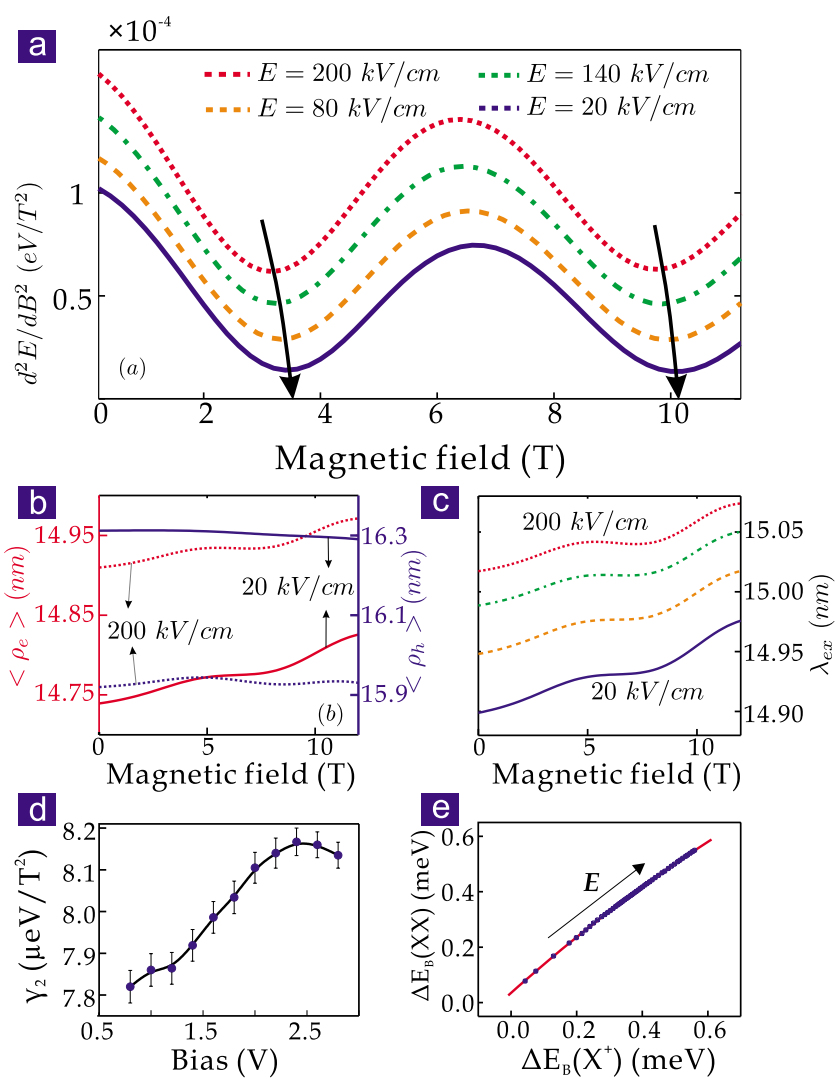

FIG. 6. (Color online) (a) Second derivative of the exciton energy of the state with $L=0$. Calculations are shown for four different values of the electric field. (b) The effective radius of the electron and the hole, defined as $\int \Psi_{L}^{*}\left(\vec{r}_{e}, \vec{r}_{h}\right) \rho_{e(h)} \Psi_{L}\left(\vec{r}_{e}, \vec{r}_{h}\right) d \vec{r}_{e} d \vec{r}_{h}$. (c) The radius $\lambda_{e x}=\left[\left(m_{h}+m_{e}\right) /\left(m_{h} /\left\langle\rho_{e}\right\rangle^{2}+m_{e} /\left\langle\rho_{h}\right\rangle^{2}\right)\right]^{0.5}$, which corresponds to the exciton radius definition for a $3 \mathrm{D}$ system. (d) The diamagnetic coefficient $\gamma_{2}$ changes with applied bias. (e) The binding energies of $\mathrm{XX}$ and $\mathrm{X}^{+}$change by nearly the same amount with external $E$ field.

to $20 \mathrm{kV} / \mathrm{cm}$, the electron is attracted to the bottom area of the ring, decreasing its effective radius, while the hole is pushed to the top of the ring and its effective radius increases. From the change in $\left\langle\rho_{e}\right\rangle$ and $\left\langle\rho_{h}\right\rangle$ alone we cannot conclude that the period of the Aharonov-Bohm oscillation decreases. Theoretical study reveals that the oscillation comes from a change in the value of the angular momentum pair $\left(l_{e}, l_{h}\right)$, not from the single electron or hole angular momentum. The period of the oscillation is not only related to the effective radius of the electron and the hole but also to their effective masses.

We find that the magnetic field at which the first transition takes place is proportional to $\hbar / e \lambda_{e x}^{2}$, where $1 / \lambda_{e x}^{2}=\left(m_{h} /\right.$ $\left.<\rho_{e}>^{2}+m_{e} /<\rho_{h}>^{2}\right) /\left(m_{e}+m_{h}\right)$. We plot $\lambda_{e x}$ as a function of magnetic field for different values of electric field in Fig. 6(c). Because the effective mass of the hole is much larger than that of the electron (also because the electron and the hole radii change within the same order), $\lambda_{e x}$ should have a similar behavior as the electron effective radius $\left\langle\rho_{e}\right\rangle$. This is clearly observed in Fig. 6(c) and $\lambda_{e x}$ decreases monotonously with decreasing electric field. As a result, the first transition takes place at a higher magnetic field when we decrease the 


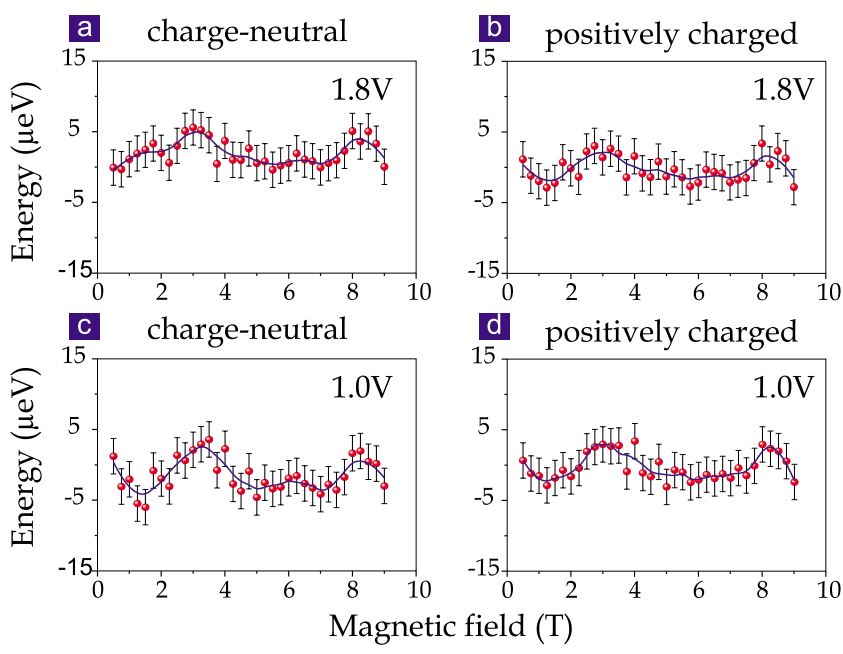

FIG. 7. (Color online) Charge-neutral and positively charged exciton complexes show similar peak energy oscillations. The data are given at $V=1.8$ and $1.0 \mathrm{~V}$. The solid line represents the smoothed data and is used as guide to the eye.

electric field [see Fig. 5(b)]. In fact, $\lambda_{e x}$ should be proportional to the diamagnetic coefficient, which explains why we observe in the experiment an increase in $\gamma_{2}$ with external bias [see Fig. 6(d)]. The explanation for the second transition is similar, although the formula for the magnetic field at which it takes place is different (also it has the dominant term $m_{h} /\left\langle\rho_{e}\right\rangle^{2}$ ), which shifts to higher magnetic field when the electric field decreases.

\section{B. Charged exciton emission}

It is worth mentioning that for the studied QRs we do not observe substantially different oscillation behaviors for charged exciton complexes. Figure 7 shows the peak energy oscillations of the charge-neutral and of the positively charged exciton complexes for the QR studied in Fig. 5. We choose $V=1.8 \mathrm{~V}$ where the charge-neutral emission intensity reaches maximum and $V=1.0 \mathrm{~V}$ where the positively charged exciton emission intensity reaches maximum (see also Fig. 8). This observation is not clearly understood yet because intuitively one expects that a charged exciton (e.g., $\mathrm{X}^{+}$) should be more sensitive to the $\mathrm{AB}$ effect than the neutral exciton. ${ }^{14}$ It might be that the oscillation period difference between the neutral and charged excitons is quite small. We propose a possible mechanism that the exciton complexes confined in our QR are in the strongly confined fewparticle regime (in other words, the exciton complexes are weakly bound) and the interparticle Coulomb interactions are perturbations to the single-particle energy. This is reasonable since in the ring geometry the ratio of Coulomb to kinetic energies is proportional to the ring radius while for small rings the quantization due to kinetic motion is strong and the effectiveness of Coulomb interaction becomes weaker., ${ }^{6,29}$ The presences of the $n$-doped layer which locates $20 \mathrm{~nm}$ below the QRs and the metal contact layer on top further reduce the Coulomb interaction. ${ }^{10}$ In fact we observe that the changes in the binding energies $\Delta E_{B}(\mathrm{XX}) \approx \Delta E_{B}\left(\mathrm{X}^{+}\right)$when
$E$ changes [Fig. 6(e)], indicating that the strongly confined few-particle picture is valid in our QRs and, the effective radii of electron and hole are very different. ${ }^{30,31}$

\section{B-dependent PL intensity}

According to the theory predictions ${ }^{6}$ and the existing experimental reports on QD ensembles, ${ }^{16,17}$ the PL intensity should also oscillates with magnetic field and the oscillation amplitudes are around 5-10\%. ${ }^{16,17}$ For the QR studied in Fig. 5, the PL intensity versus magnetic field are plotted at several different bias in Fig. 8 [note that Fig. 8(a) is subset data of Fig. 5(c)]. With increasing forward bias, the PL intensity shows a rich pattern, other than simply quenching as shown in Fig. 4(e). The general trend is marked by red solid lines in Fig. 8. For the charge-neutral exciton complex the variation in PL intensity with increasing $B$ field is small at low-voltage range (below 1.4 V) [see Fig. 8(a)]. At $1.8 \mathrm{~V}$, where the PL intensity reaches an overall maximum, the intensity shows clear oscillation with $B$ field, with a minima at $\sim 5.5 \mathrm{~T}$. This corresponds well with the PL energy oscillations shown in Fig. 7(a). Beyond 2.2 V the overall PL intensity slightly decreases with bias and the PL signal is not fully recovered at high magnetic field, as we see for QRs that are not embedded in FET structure [Fig. 4(e)]. In Fig. 8(a) we also observe intensity maxima at low magnetic fields, it changes from $\sim 3.5 \mathrm{~T}$ at $0.8 \mathrm{~V}$ to $\sim 2.6 \mathrm{~T}$ at $2.8 \mathrm{~V}$. This, again, corresponds quite well with the PL energy oscillation shifts observed in Fig. 5(b).

The PL intensity of the positively charged exciton shows similar behavior, see Fig. 8(b). At low-voltage range (below $1.0 \mathrm{~V})$ the PL intensity slightly increases with increasing $B$ field, which is slightly different from Fig. 8(a). At $\sim 1.0 \mathrm{~V}$ where the overall intensity saturates, oscillations can clearly be observed. At high-voltage range (above $1.0 \mathrm{~V}$ ), there is also significant PL quenching at high magnetic fields.

In the experiments the vertical electrical field plays an important role. After being embedded in FET structure, the QRs experience an internal piezoelectric field which separates the electron and the hole. The QCSE experiment implies that the hole wave function lies above that of the electron at zero electric field. It is reasonable because an indiumrich core presents in our structure which has large uniaxial strain and provides strong confinement potential on the hole. ${ }^{32}$ As the external electrical field is increased, the hole (electron) is attracted to the bottom (apex) of the QR, increasing the overlap between electron and hole wave functions. This explains why we see the overall enhancement of the PL intensity with increasing bias in Fig. 8. When the vertical electron-hole overlap reaches the maximum $[1.8 \mathrm{~V}$ in Fig. 8(a) and $1.0 \mathrm{~V}$ in Fig. 8(b)], we see clear AB-type quantum interference effect in the PL intensity.

A previous study by Grochol et al. ${ }^{7}$ revealed that even a slightly eccentric ring geometry can smooth the oscillations and renders the total angular momentum a nonwell-defined quantum number. The selection rules are only strictly applicable in a system with perfect rotational symmetry, and at high magnetic fields an oscillator strength transfer is expected between excitonic bright states and dark states, this 
a charge-neutral

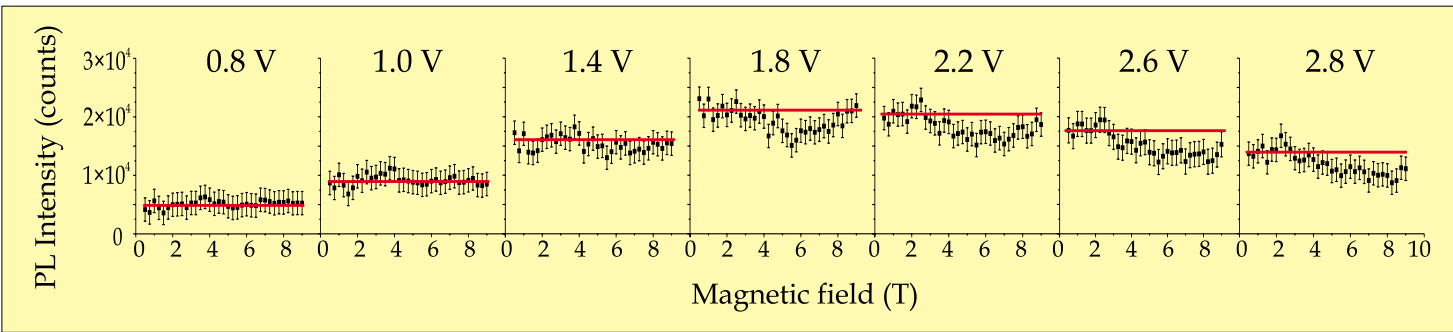

b positively charged

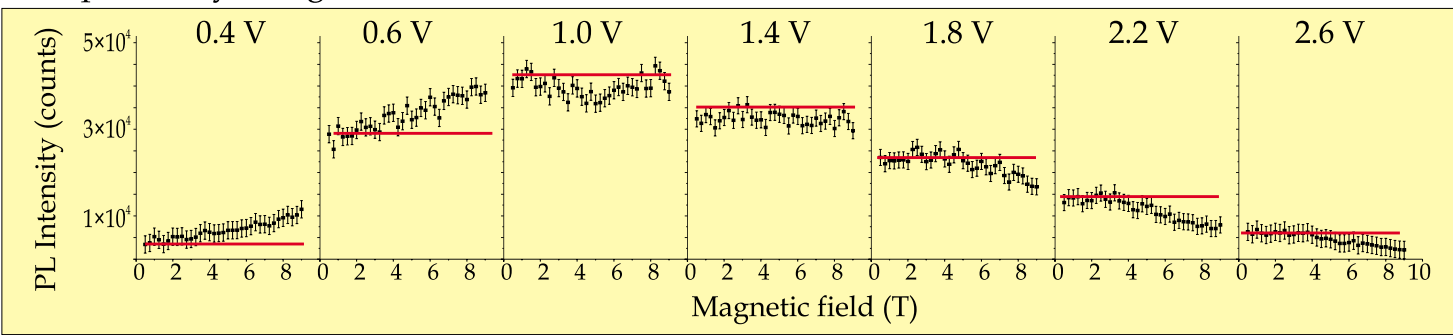

FIG. 8. (Color online) PL intensity for the (a) charge-neutral and (b) positively charged exciton complexes plotted versus $B$ field at several different bias. The solid lines mark the general shift of the PL intensity with increasing bias.

explains why the PL intensity quenches with increasing magnetic fields. However, we are not able to interpret the PL enhancement of positively charged exciton at high magnetic fields in the low electrical field range.

\section{CONCLUSION}

In conclusion, we present data on the magnetophotoluminescence study of a single self-assembled semiconductor nanoring which is fabricated by $\mathrm{AsBr}_{3}$ in situ etching. The exciton complexes in these volcano-shape QRs are in the strong confinement regime and the effective radii of the electron and hole wave functions are different, giving rise to a nonzero net magnetic flux, hence the observation of oscillations in the exciton PL energy and PL intensity in a magnetic field. The oscillations can be controlled by applying a vertical electric field which modifies the electron and hole radii. Although the experimental features are not perfectly under- stood yet, we propose that the main results can be explained by a microscopic model of how a single exciton behaves under the combination of magnetic and electric fields. We expect that the $A B$ effect for a single exciton is quite small, thus higher resolution spectroscopy methods are needed. Further experiments will also focus on the utilization of a lateral electric field to further increase the polarization of an exciton, hence the enhancement of the observed AB-type effect.

\section{ACKNOWLEDGMENTS}

We acknowledge L. P. Kouwenhoven and Z. G. Wang for support, L. Wang, V. Fomin, S. Kiravittaya, M. Tadic, WenHao Chang, I. Sellers, A. Avetisyan, and C. Pryor for fruitful discussions and the financial support of NWO (VIDI), the CAS-MPG program, the DFG (FOR730), BMBF (Grant No. 01BM459), NSFC (Grant No. 60625402), and Flemish Science Foundation (FWO-Vl). Access to the TEM of B. Rellinghaus is acknowledged. *f.ding@ifw-dresden.de

†v.zwiller@tudelft.nl

${ }^{1}$ Y. Aharonov and D. Bohm, Phys. Rev. 115, 485 (1959).

${ }^{2}$ S. Popescu, Nat. Phys. 6, 151 (2010).

${ }^{3}$ A. G. Aronov and Y. Sharvin, Rev. Mod. Phys. 59, 755 (1987).

${ }^{4}$ S. Zaric, G. N. Ostojic, J. Kono, J. Shaver, V. C. Moore, M. S. Strano, R. H. Hauge, R. E. Smalley, and X. Wei, Science 304, 1129 (2004).

${ }^{5}$ A. Fuhrer, S. Luscher, T. Ihn, T. Heinzel, K. Ensslin, W. Wegscheider, and M. Bichler, Nature (London) 413, 822 (2001).

${ }^{6}$ A. O. Govorov, S. E. Ulloa, K. Karrai, and R. J. Warburton, Phys. Rev. B 66, 081309 (2002).

${ }^{7}$ M. Grochol, F. Grosse, and R. Zimmermann, Phys. Rev. B 74,
115416 (2006)

${ }^{8}$ A. Lorke, R. J. Luyken, A. O. Govorov, J. P. Kotthaus, J. M. Garcia, and P. M. Petroff, Phys. Rev. Lett. 84, 2223 (2000).

${ }^{9}$ N. A. J. M. Kleemans et al., Phys. Rev. Lett. 99, 146808 (2007).

${ }^{10}$ L. G. G. V. Dias da Silva, S. E. Ulloa, and T. V. Shahbazyan, Phys. Rev. B 72, 125327 (2005).

${ }^{11}$ R. A. Römer and M. E. Raikh, Phys. Rev. B 62, 7045 (2000).

${ }^{12}$ A. Chaplik, JETP Lett. 62, 900 (1995).

${ }^{13}$ M. Tadić, N. Čukarić, V. Arsoski, and F. Peeters, arXiv:1003.0467 (unpublished).

${ }^{14}$ M. Bayer, M. Korkusinski, P. Hawrylak, T. Gutbrod, M. Michel, and A. Forchel, Phys. Rev. Lett. 90, 186801 (2003).

${ }^{15}$ E. Ribeiro, A. O. Govorov, W. Carvalho, and G. Medeiros- 
Ribeiro, Phys. Rev. Lett. 92, 126402 (2004).

${ }^{16}$ I. R. Sellers, V. R. Whiteside, I. L. Kuskovsky, A. O. Govorov, and B. D. McCombe, Phys. Rev. Lett. 100, 136405 (2008).

${ }^{17}$ M. D. Teodoro et al., Phys. Rev. Lett. 104, 086401 (2010).

${ }^{18}$ A. V. Maslov and D. S. Citrin, Phys. Rev. B 67, 121304 (2003).

${ }^{19}$ A. M. Fischer, V. L. Campo, Jr., M. E. Portnoi, and R. A. Römer, Phys. Rev. Lett. 102, 096405 (2009).

${ }^{20}$ A. A. High, E. E. Novitskaya, L. V. Butov, M. Hanson, and A. C. Gossard, Science 321, 229 (2008).

${ }^{21}$ F. Ding, L. Wang, S. Kiravittaya, E. Müller, A. Rastelli, and O. G. Schmidt, Appl. Phys. Lett. 90, 173104 (2007).

${ }^{22}$ R. J. Warburton, C. Schaflein, D. Haft, F. Bickel, A. Lorke, K. Karrai, J. M. Garcia, W. Schoenfeld, and P. M. Petroff, Nature (London) 405, 926 (2000).

${ }^{23}$ T.-C. Lin, C.-H. Lin, H.-S. Ling, Y.-J. Fu, W.-H. Chang, S.-D. Lin, and C.-P. Lee, Phys. Rev. B 80, 081304 (2009).

${ }^{24}$ R. J. Young, R. M. Stevenson, A. J. Shields, P. Atkinson, K. Cooper, D. A. Ritchie, K. M. Groom, A. I. Tartakovskii, and M. S. Skolnick, Phys. Rev. B 72, 113305 (2005).

${ }^{25}$ R. M. Stevenson, R. J. Young, P. See, D. G. Gevaux, K. Cooper,
P. Atkinson, I. Farrer, D. A. Ritchie, and A. J. Shields, Phys. Rev. B 73, 033306 (2006).

${ }^{26}$ K. Kowalik, O. Krebs, A. Lemaître, S. Laurent, P. Senellart, P. Voisin, and J. A. Gaj, Appl. Phys. Lett. 86, 041907 (2005).

${ }^{27}$ T. Kuroda, T. Belhadj, T. Mano, B. Urbaszek, T. Amand, X. Marie, S. Sanguinetti, K. Sakoda, and N. Koguchi, Phys. Status Solidi B 246, 861 (2009).

${ }^{28}$ V. M. Fomin, V. N. Gladilin, J. T. Devreese, N. A. J. M. Kleemans, M. Bozkurt, and P. M. Koenraad, Phys. Status Solidi B 245, 2657 (2008).

${ }^{29}$ M. Korkusiński, P. Hawrylak, and M. Bayer, Phys. Status Solidi B 234, 273 (2002).

${ }^{30}$ F. Ding et al., Phys. Rev. Lett. 104, 067405 (2010).

${ }^{31}$ M.-F. Tsai, H. Lin, C.-H. Lin, S.-D. Lin, S.-Y. Wang, M.-C. Lo, S.-J. Cheng, M.-C. Lee, and W.-H. Chang, Phys. Rev. Lett. 101, 267402 (2008).

${ }^{32}$ R. J. Warburton, C. Schulhauser, D. Haft, C. Schäflein, K. Karrai, J. M. Garcia, W. Schoenfeld, and P. M. Petroff, Phys. Rev. B 65, 113303 (2002). 